\title{
35. \\ Information Modeling for Product Lifecycle Management
}

\author{
Q.Shu and Ch.Wang \\ College of Mechanical Engineering \& Automation, Northeastern University, 110004, \\ Shenyang, PR China \\ E-mail:shu_qilin@mail.neu.edu.cn
}

\begin{abstract}
Product lifecycle modelling is to define and represent product lifecycle data and to maintain data interdependencies. This paper presents a framework of product lifecycle model that comprises three parts: product information model, process model based on product life cycle, and extended enterprise resource model. Further, the relationship and formation of product models at different stages are described. Finally, an integrated information architecture is proposed to support interoperability of distributed product data sources.
\end{abstract}

\section{INTRODUCTION}

In today's distributed manufacturing environment, how to manage the product data and distribute it to the right people who need it is critical to the success of an enterprise. At present, the existing systems such as CAx, ERP, PDM, SCM, CRM, eBusiness etc. are just solutions for some stages of a product lifecycle, which is difficult to support an enterprise to operate efficiently. Developing a complex product requires not only all collaboration among departments of an enterprise but also cooperation of other enterprises in different regions. To effectively allocate enterprise's resources and harmonize business activities, enterprises must integrate all information relevant to various stages of a product lifecycle and all the information throughout a product lifecycle can be accessed by everyone associated with its design, creation, sale, distribution, and maintenance. Now enterprises press for an information architecture to implement product lifecycle management. The product lifecycle modelling technology supports description, transmitting and sharing of data in distributed environment. Therefore, the product lifecycle modelling technology and related information management systems are taken seriously by academia and industries.

Many researchers have conducted study on product models at different stages of product lifecycle. Bidarra and Brosvoort described product structures and its function information by building engineering design model based on semantic feature method (Biddarra and Brosvoort, 2000). Jiao et al. proposed a generic billof-materials-and-operations for high-variety production management to integrate data of product structure and manufacturing information, which support engineering changes and transactions of customers' orders (Jiao et al., 2000). Simon et al. presented a modelling technology of the life cycle of products with data acquisition features for washing machines based on a microcontroller and non-volatile memory (Simon et al., 2001). This life cycle model with data acquisition features supported 
activities of design, marketing and servicing as well as end-of-life. Shehab et al. proposed a manufacturing cost model for concurrent product development to help inexperienced designers evaluate the manufacturing cost of products at the conceptual design stage (Shehab et al., 2001).

To remain competitive and to respond the market rapidly, enterprises have to cooperate effectively as the form of extended enterprise to develop complex products. An extended enterprise comprises an Original Equipment Manufacturer (OEM), its supply chain, subsidiaries, consultants, and partners affiliated with the life cycle of a particular family of products. Rezayat identified a majority of the components needed to implement the E-Web and showed how E-Web can provide support for everyone associated with a product during its life cycle (Rezayat, 2000).

Manufacturing paradigms such as agile virtual enterprise, Internet-based manufacturing, and collaborative manufacturing require creating a distributed environment that enables integrated product, process, and protocols development in order to manage and maintain the distributed product data as a whole. Product lifecycle model provides a conceptual mapping mechanism to associate with data of product design, manufacturing, quality, cost, sales, operation, and service. The model also supports the access and operation of distributed data. Xue thinks that product lifecycle model should comprise a series of models at different stages and these models can be generated automatically by a method (Xue, 1999). Zhang and Xue proposed product lifecycle modelling method based on distributed databases, which defined the relationship between data located in different regions and activities by using and/or graph (Zhang and Xue, 2001). Tianfield built an advanced lifecycle model for complex product development (Tianfield, 2001). The model comprised concurrent engineering, production schedule control, virtual prototype, and enterprise information integration. Now it is commonly accepted that the Web is a major enabler in this regard due to its open standards, ease of use, and ubiquity, so some researchers proposed or implemented product lifecycle information infrastructure based on the Internet (Rezayat, 2000; Wang, 2001).

\section{FRAMEWORK OF PRODUCT LIFECYCLE MODEL}

Now $50-80 \%$ of all components in products from Original Equipment Manufacturers (OEMs) are fabricated by outside suppliers, and this trend is expected to continue (Rezayat, 2000). During a product lifecycle, the product data are generated and operated in multiple business processes among different departments in the extended enterprise. So, how to integrate these data and processes to support the product lifecycle activities is becoming very important. In this paper we present an information framework to describe all processes and activities during the lifecycle of a product by building integrated product information model, process model based on product life cycle, and extended enterprise resource model as shown in Figure 1.

It is impossible to describe all activities and processes during the product lifecycle only using a single model because of complexity of product developing and using process. Therefore, it is necessary to build a group of models according to different stages and aspects. We divide the product lifecycle into five stages, which are requirement analysis stage, conceptual design stage, engineering design stage, manufacturing stage, and service \& support stage. Consequently, we propose that the product lifecycle model comprises requirement analysis model, conceptual 
design model, engineering design model, manufacturing model, and service \& support model, and all related data and documents which stored in the distributed databases form those models through logical mapping. The product requirement analysis model represents customer's needs and interests in customer's language, and this model relates to subjective origins of a product. The product conceptualization model is a conceptual definition of a product including its functions, implementing theory, basic structure information, rough costing, and some key components' design etc. The product engineering design model is the core of product lifecycle model, which depicts both geometric and engineering semantic information by using $\mathrm{CAD}$ files and related data. The product manufacturing model describes the information relevant to the processes of fabrication and assembly such as material, manufacturing process planning, and assembly sequences etc. The service \& support model is about the information of products' delivery state, installation, operation, training, maintenance, diagnosis, etc.

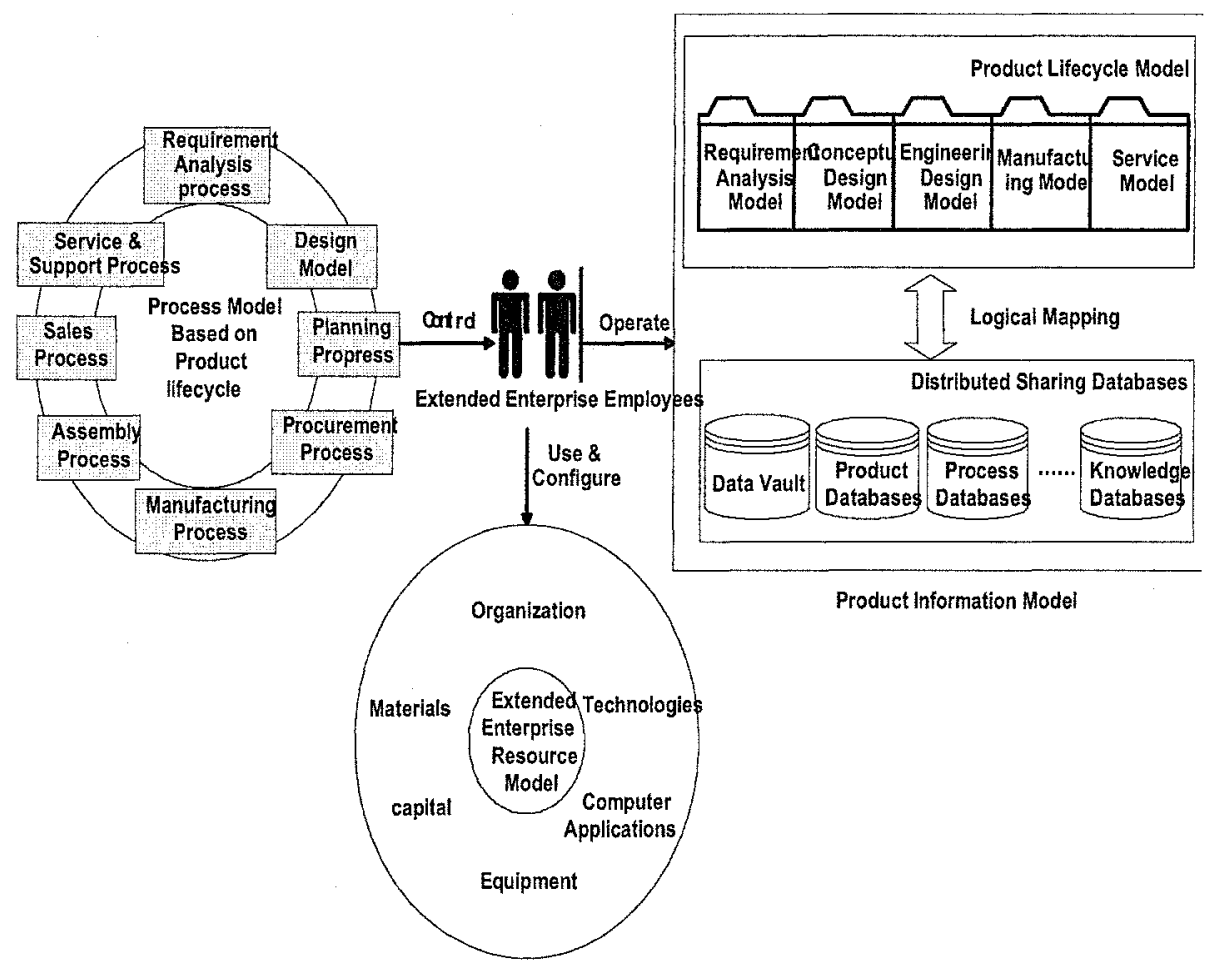

Figure 1 Framework of Product Lifecycle Model

The process model based on product lifecycle enhances and extends the functions of workflow in PDM systems, and it describes the relationship among data, applications, enterprise resources, organizations, and employees in various stages during the product formation. This model supports dynamical process definitions of product data, projects management, and process scheduling \& planning. The process model comprises macro processes and various micro processes. The macro processes during a product lifecycle include the processes of requirement analysis, 
engineering design, process planning, procurement, manufacturing, assembly, sale, and service \& support. However, the micro processes exits in those macro processes, e.g. the micro process of the examining and approving in the macro processes of both design and process planning. The process model guarantees the security and effectiveness of all product data by controlling the right operation of data by right people, and coordinating the relationship of various departments in the extended enterprise.

The extended enterprise resource model provides resource configuration for various stages of the product lifecycle. It describes the resource structures in the extended enterprise and relationship among the different resources. The extended enterprise resource model primarily comprises organizations, technologies, materials, capital, equipment, and various computer applications etc. In a manufacturing enterprise, employees are organized to perform corresponding tasks by a strict organizing structure and a set of rules, so they are the most important resource in an enterprise. At the same time the employees in the extended enterprises who use and configure the enterprise resources, operate data and documents in the product lifecycle model are guided by the process model based on the product lifecycle. The relationship among the process model, resource model, and product information model is shown in Figure 1.

\section{RELATIONSHIP AND FORMATION OF PRODUCT MODELS AT DIFFERENT STAGES}

A product is composed of parts and/or components, and a component is composed of parts and/or sub-assemblies. Therefore, its structure tree describes the product structure. The product structure should be different when different departments at different stages in the product lifecycle operate it. The models in different stages are built by associating all related data, documents, and the relationship among them with relevant nodes in the product structure tree. The product requirement analysis model can acquire and express customers' needs, realize the conversion of product information from customer view into designer view. There are three levels to build the product requirement analysis model: acquire customers' requirement information, express the requirement information, and analyze the requirement information. The customers' requirement information can be acquired through communication with customers by various means, and finally the primal object tree is formed. This primal object tree describes the outline of product from customer's view, so it only expresses the basic requirements and expectations of customers.

At the conceptual design stage, the product model comprises two parts: product function model and product conceptualization model. First designers map the primal object tree into product function tree from the view of technology implementation. For example, customers need to acquire information from outside through their computers, so designers can map this requirement into the function of access to the Internet. Then designers may further map every node in the product function tree into one or more physical modules (parts or components), e.g. mapping the function of access to the Internet into physical module of network card or modem. Therefore, the product requirement analysis model can be converted into the product conceptualization model in this way. 
At the engineering design stage, designers form a generic product structure through analyzing, computing, simulating, and testing by making full use of the product conceptualization model and considering product varieties, design constraints, and resources limitations etc.

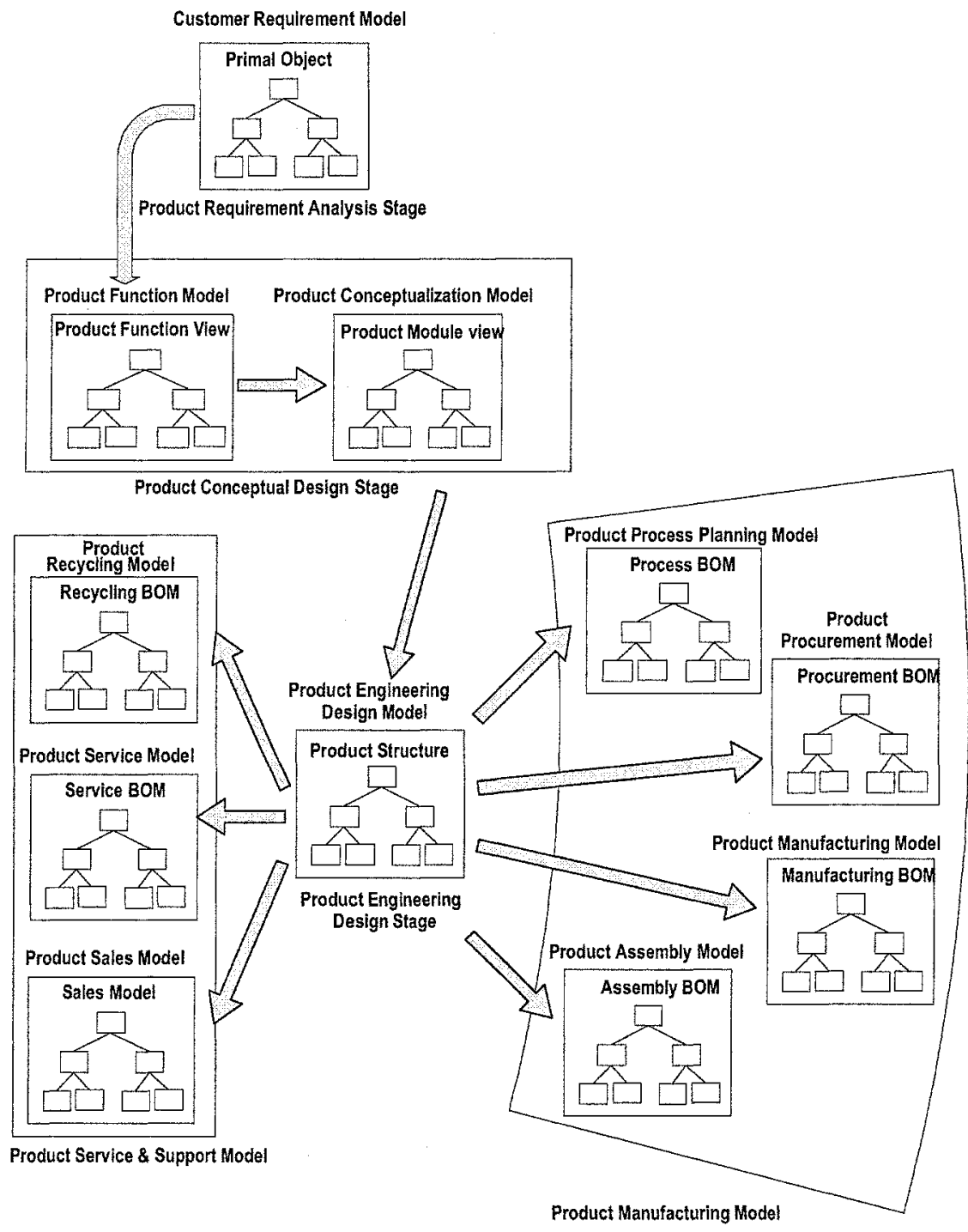

Figure 2 Relationships and Formation of Product Models at Different Stages

Every node in the generic product structure tree should be associated with related documents and data such as CAD files, design reports, analysis reports, test data, using materials, working space, kinematic parameters, and working precision etc. With these, we form the product engineering design model based on the product structure. The product engineering design model is the core staged model of the product lifecycle model, from which the manufacturing model and the service \& 
support model can be derived through reconfiguring the engineering BOM and adding additional related information by corresponding business department of the extended enterprise. For example, the process planning model at the manufacturing stage is built by reconfiguring the engineering BOM into process BOM and associating relevant process planning information with each node in the process BOM. In the same way the procurement model, the manufacturing model, and the assembly model can be formed based on the engineering design model.

The product service \& support model collects all information about sale, transportation, installation, operation, maintenance, and recycling, which comprises three sub-models: the sale model, the service model, and the recycling model. All these information including various digital documents, CAD files (2D/3D), multimedia files (videos/audios/ animation) is associated with related nodes in the BOMs (sale BOM, service BOM, and recycling BOM). All these BOMs (sale BOM, service $B O M$, and recycling $B O M$ ) are reconfigured based on the product engineering design model (engineering $\mathrm{BOM}$ ). The relationship and formation of product models at different stages is shown in Figure 2.

\section{INTEGRATED INFORMATION FRAMEWORK OF PRODUCT LIFECYCLE MODEL}

The product lifecycle management (PLM) system is a network-oriented manufacturing system, which comprises many sub-systems. These sub-systems are located in a distributed heterogeneous environment. Therefore, we must find effective methods for communication and sharing of information, especially those related to design and manufacturing, throughout the entire enterprise and the supply chain. The technologies that support such methods must be able to deal with distributed environments and databases, must ensure reliability and security, and must be practical. We present an integrated information framework of the product lifecycle model based on J2EE to realize the goal of providing the right information to the right person at the right time and in the right format anywhere within the extended enterprise.

As shown in Figure 3, the information framework of the product lifecycle model is composed of three tiers: application tier, service tier, and data tier. There are five application agents according to five stage models of the product lifecycle model in the application tier. They are: requirement agent, concept agent, design agent, manufacturing agent, and service agent. The five agents run in two containers, one is Applet container and another is application container. There are two ways to access information for every agent, that is Web-based way and non-Web way. For nonWeb way, there is a client application to run on local computers, and for Web-based way the browser can download Web pages and Applets to client computers. The requirement agent, concept agent, and service agent are usually based on Web and they are implemented by Applets. However, the design agent and manufacturing agent are always run on Intranet within an enterprise, so they are implemented by client application instead of basing on Web.

The application agents access the basic service agents such as graphics agent, coordinating agent, security agent, query agent, and resource agent etc. in the service tier through various protocols (e.g. TCP/IP, SOAP, and IIOP etc.). The graphics agent displays the geometries $(2 \mathrm{D} / 3 \mathrm{D})$ of products. The security agent guarantees 
the security of the whole product lifecycle management system by verifying identification and authorizing operation etc. Among these basic service agents, the query agent is a very important one, which responds to the querying requirements of users and provides the desired results to them by automatically searching all information sources in the product lifecycle model. The resource agent manages all system resources according to the request of other agents in order to avoid conflict between agents. These service agents are implemented based on Web container and EJB container. Web components provide Web services, which can be JSP pages or Servlets. Servlets are the classes of Java, which can dynamically respond to the request. However, JSP pages are based on text files that contain static texts and a fragment of Java program. When JSP pages are loaded, the Servlet executes the fragment of Java program and returns the results. EJB components are enterprise business components, which perform service functions of enterprise business such as security agent, resource agent, and coordinating agent etc.

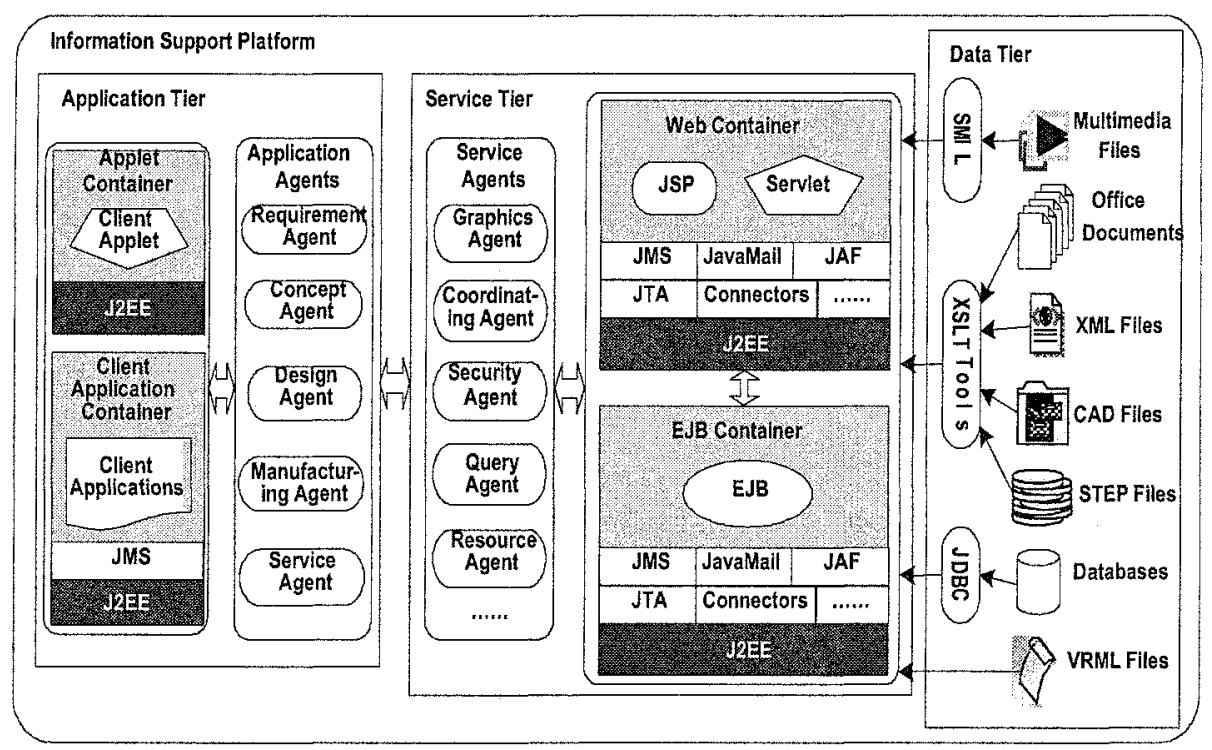

Figure 3 A Support Framework of Product Lifecycle Model

In the data tier, all the data are stored in physical media as various forms. To effectively manage and browse these data through the Internet, we convert the nonstructured data with different formats into XML files by using XSLT tools. These files with different formats include office documents, CAD data, STEP files, and multimedia files etc. The structured data in the relational databases can be accessed through JDBC. The product lifecycle information framework based on the above three-tier architecture has the advantages of interoperation, reusability, and platform independence. The application agents in the application tier and the service agents in the service tier can be deployed in any servers compatible with J2EE, so in this way the PLM system can be established quickly and conveniently to meet enterprises' requirement for product lifecycle management. All data sources in the data tier can be stored in different locations and they can be managed through Web by various agents in the service tier. There is a great advantage to integrate and manage all 
partners' information during the product lifecycle. All PLM system components based on J2EE are reusable and extendable. They can be reused not only in the same system but in different systems (e.g. ERP, CRM etc.) as well.

\section{CONCLUSION AND FURTHER WORK}

Nowadays, information is the most important factor for a manufacturing enterprise. Product lifecycle information model helps a manufacturer to make decisions about management, design, production, operation, maintenance, and repair. The product lifecycle can be divided into five stages: requirement analysis, conceptual design, engineering design, manufacturing, and service \& support. To integrate all information of a product lifecycle and support networked manufacturing mode, we present a framework of product lifecycle model that comprises three parts: product information model, process model based on product life cycle, and extended enterprise resource model. Further, we describe the relationship and evolvement of product models at different stages. Finally, an integrated information architecture is proposed to support interoperability of distributed product data sources.

\section{REFERENCES}

Chengen Wang, Chengbin Chu, Chaowan Yin (2001) Implementation of Remote Robot Manufacturing over Internet. Computers In Industry 45(3), 215-229

Deyi Xue (1999) Modelling of Product Life-cycle Knowledge and Data for an Intelligent Concurrent Design Systems, in Knowledge Intensive Computer Aided Design, Finger, S., Tomiyama, T., and Mantyla. M. (eds.): Kluwer Academic Publishers. pp 117-142

E.M.Shehab, H. S. Abdalla (2001) Manufacturing Cost Modelling for Concurrent Product Development, Robotics and Computer Integrated Manufacturing 17:341-353

F.Zhang, D.Xue (2001) Optimal Concurrent Design Based upon Distributed Product Development Life-cycle Modelling. Robotics and Computer Integrated Manufacturing 17, 469-486

Huaglory Tianfield (2001) Advanced Life-cycle Model for Complex Product Development via Stage-Aligned Information-substitutive Concurrency and Detour. Int Journal of Computer Integrated Manufacturing 14(3), 281-303

Jianxin Jiao, Mitchell M.Tseng $\square$ Qinhai Ma and Yi Zou (2000) Generic Bill-ofMaterials-and-Operations for High-Variety Production Management, Concurrent Engineering: Research and Applications 8(4), 297-321

Mattew Simon, Graham Bee $\square$ Philip Moore, Jun-Sheng Pu, Changwen Xie (2001) Modelling of the Life Cycle of Products with Data Acquisition Features, Computers in Industry 45:111-122

M.Rezayat (2000) The enterprise-web portal for life-cycle support, Computer-Aided Design 32:85-96

M. Rezayat (2000) Knowledge-based Product Development Using XML and KCs, Computer-aided Design 32, 299-309

R.Bidarra, W.F.Brosvoort (2000) Semantic Feature Modelling. Computer-Aided Design 32:201-225 